\title{
Semianalytical analysis of shear walls with the use of discrete-continual finite element method. Part 1: Mathematical foundations
}

\author{
Pavel Akimov ${ }^{1,2^{*}}$, Mojtaba Aslami ${ }^{3}$, Marina Mozgaleva ${ }^{1}$, and Zhiuli Mskhalaya ${ }^{1}$ \\ ${ }^{1}$ National Research Moscow State University of Civil Engineering, Department of Applied \\ Mathematics, 26, Yaroslavskoe Shosse, 129337 Moscow, Russia \\ ${ }^{2}$ Russia Academy of Architecture and Construction Sciences, Presidium, 24, ul. Bolshaya Dmitrovka, \\ 107031 Moscow, Russia \\ ${ }^{3}$ Fasa university, Daneshjou blvd, Fasa, Fars province, Iran
}

\begin{abstract}
The distinctive paper is devoted to the two-dimensional semianalytical solution of boundary problems of analysis of shear walls with the use of discrete-continual finite element method (DCFEM). This approach allows obtaining the exact analytical solution in one direction (so-called "basic" direction), also decrease the size of the problem to onedimensional common finite element analysis. The resulting multipoint boundary problem for the first-order system of ordinary differential equations with piecewise constant coefficients is solved analytically. The proposed method is rather efficient for evaluation of the boundary effect (such as the stress field near the concentrated force). DCFEM also has a completely computer-oriented algorithm, computational stability, optimal conditionality of resultant system and it is applicable for the various loads at an arbitrary point or a region of the wall.
\end{abstract}

\section{Introduction}

Problems of structural analysis normally lead to complex two-dimensional and threedimensional systems of equations, and solutions of these systems can be obtained only by numerical approaches [1-3]. Generally it is impossible to find the correct analytical (exact) solution $[4,5]$ while corresponding experimental researches are more expensive and noncomplete. Recent developments in computer industry and mathematics resulted in emerging new branches in computational mechanics under the name of semi-analytical or discretecontinual methods [6-11]. Semi-analytical approaches allow obtaining the solution in correct analytical form, which is more efficient, especially in the zones of boundary effects. Results have normally fast variational behaviour there. Therefor accuracy and convergence of solutions obtained by numerical methods are often heavily dependent on the type of selected basis functions (shape functions) for approximating of unknowns, and the number of discretization nodes (elements) [12,13]. Convergence in the areas of boundary effects, located near the concentered forces and stress concentrations (i.e. in the most critical areas)

* Corresponding author: pavel.akimov@gmail.com 
are very slow for numerical approaches and weakly depends on the number of elements. Even, for example, if the convergence for displacements is relatively high, it is much smaller for internal strains and stresses. Besides, preliminary simplified analytical research of complicated problem can be very useful for understanding of solution properties or behaviour of structure. Applications of discrete-continual methods provides substantial reduction of size of problems, especially for multidimensional analysis. Fields of the applications of discrete-continual method comprises structures with regular (constant or piecewise constant) physical and geometrical parameters (characteristics) in one (so-called "basic") direction (for examples, tall buildings, tunnels, railroads, dams, foundations, etc.). Exact analytical evaluation of the behaviour (stress-strain state) of such structure in the basic direction is of paramount importance. Discrete-continual methods provide correct analytical solution of the considering problem along basic direction of structure and numerical (for instance, finite element) approximation along other directions. As a result, more accurate solutions can be obtained, especially in zones of boundary effect.

The distinctive paper is devoted to two-dimensional (plane stress condition) semianalytical analysis of shear wall with the use of discrete-continual finite element method (DCFEM) $[14,15,16]$. It allows obtaining the exact analytical solution in one direction and decreasing the size of the problem to conventional one-dimensional finite element analysis.

\section{Formulation of the problem}

Shear walls, are vertical cantilevered lateral-force-resisting elements, which are normally used as supports for the roof and floor diaphragms, or for the transfer of loads into diaphragms (where the wall cannot continue to the foundation $[17,18]$ ). Shear walls can be full-length walls or short wall sections that can occur at any location along a line of lateral force resistance. When a diaphragm is loaded by wind or seismic forces, the loads are transferred from the diaphragm into the top of the supporting shear walls or other boundary elements at each end of the diaphragm. Shear walls resist in-plane loads that are applied along its height. In many cases, shear walls can be simplified into several two-dimensional problem. Considering this point, a plane stress condition is used for the simulation within DCFEM. The common shear walls have a rectangular or a piecewise rectangular shape with long regular length and therefor can be solved efficiently by this method. Due to substantial height of the shear walls, the vertical direction is used for the analytical solution.

\subsection{Continual operational formulation of the problem}

Let $x_{2}$ be coordinate corresponding to basic direction; $x_{2, k}^{b}, k=1, \ldots, n_{k}$ are coordinates of boundary points i.e. cross-sections (including points of changes in physical and geometrical parameters). Operational formulation of corresponding resultant multipoint boundary problem of two-dimensional theory of elasticity [19] at extended domain [20], embordering considering structure, within DCFEM has the form:

$$
\begin{aligned}
& \left\{\begin{array}{l}
\mathbf{U}_{k}^{\prime}=\widetilde{L}_{k} \mathbf{U}_{k}+\mathbf{S}_{k}, \quad x_{3} \in\left(x_{3, k}^{b}, x_{3, k+1}^{b}\right), \quad k=1, \ldots, n_{k}-1 \\
\widetilde{B}_{k}^{-} \mathbf{U}_{k-1}\left(x_{3, k}^{b}-0\right)+\widetilde{B}_{k}^{+} \mathbf{U}_{k}\left(x_{3, k}^{b}+0\right)=\widetilde{\mathbf{g}}_{k}^{-}+\widetilde{\mathbf{g}}_{k}^{+}, k=2, \ldots, n_{k}-1 \\
\widetilde{B}_{1}^{+} \mathbf{U}_{1}\left(x_{3,1}^{b}+0\right)+\widetilde{B}_{n_{k}}^{-} \mathbf{U}_{n_{k}-1}\left(x_{3, n_{k}}^{b}-0\right)=\widetilde{\mathbf{g}}_{1}^{+}+\widetilde{\mathbf{g}}_{n_{k}}^{-},
\end{array}\right. \\
& \widetilde{L}_{k}=\left[\begin{array}{cc}
0 & E \\
L_{k, v v}^{-1}\left(L_{k, u u}+C_{k}\right) & L_{k, v v}^{-1} \widetilde{L}_{k, u v}
\end{array}\right] ; \mathbf{S}_{k}=-\left[\begin{array}{c}
0 \\
L_{k, v}^{-1} \widetilde{\mathbf{F}}_{k}
\end{array}\right] ; \quad \mathbf{U}_{k}=\left[\begin{array}{c}
\mathbf{u}_{k} \\
\mathbf{v}_{k}
\end{array}\right] ;
\end{aligned}
$$




$$
\begin{gathered}
L_{k, v v}=\left[\begin{array}{cc}
\bar{\mu}_{k} & 0 \\
0 & \bar{\lambda}_{k}+2 \bar{\mu}_{k}
\end{array}\right] ; \quad L_{k, u v}=\left[\begin{array}{cc}
0 & \partial_{1}^{*} \bar{\lambda}_{k} \\
\partial_{1}^{*} \bar{\mu}_{k} & 0
\end{array}\right] ; \quad L_{k, u v}=\partial_{1}^{*}\left[\begin{array}{cc}
\bar{\lambda}_{k}+2 \bar{\mu}_{k} & 0 \\
0 & \bar{\mu}_{k}
\end{array}\right] \partial_{1} ; \\
C_{k}=\left(\theta_{k}+\delta_{\Gamma, k}\right)\left[\begin{array}{cc}
c_{k, 1} & 0 \\
0 & c_{k, 2}
\end{array}\right] ; \quad \theta_{k}(x)=\left\{\begin{array}{cc}
1, & x \in \Omega_{k} \\
0, & x \notin \Omega_{k} ;
\end{array} \quad \delta_{\Gamma, k}(x)=\frac{\partial \theta_{k}}{\partial \mathbf{n}_{k}} ; \quad \widetilde{\mathbf{F}}_{k}=\theta_{k} \mathbf{F}_{k}+\delta_{\Gamma, k} \mathbf{f}_{k} ;\right. \\
\widetilde{L}_{k, u v}=L_{k, u v}-L_{k, v u} ; \quad L_{k, v u}=L_{k, u v}^{*} ; \quad \mathbf{v}_{k}=\partial_{2} \mathbf{u}_{k} ; \quad \mathbf{U}_{k}^{\prime}=\partial_{2} \mathbf{U}_{k} ;
\end{gathered}
$$

$\Omega$ is the domain, occupied by structure; $x=\left(x_{1}, x_{2}\right) ; x_{1}, x_{2}$ are coordinates $\left(x_{2}\right.$ corresponds to basic dimension); $l_{2}$ is the length of structure along basic dimension, $x_{2} \in\left[0, l_{2}\right] ; \Omega_{k}, k=1, \ldots, n_{k}-1$ are corresponding fragments of domain $\Omega$ with boundaries $\Gamma_{k}$, obtained by separation from domain $\Omega$ by cross-sections $x_{2}=x_{2, k}^{b}$ and $x_{2}=x_{2, k+1}^{b} ; \quad \omega_{k}, k=1, \ldots, n_{k}-1$ are extended domains, embordering fragments $\Omega_{k}, k=1, \ldots, n_{k}-1 ; \theta_{k}=\theta_{k}\left(x_{1}, x_{2}\right)$ is the characteristic function of domain $\Omega_{k}$; $\delta_{\Gamma, k}=\delta_{\Gamma, k}\left(x_{1}, x_{2}\right)$ is the delta-function of border $\Gamma_{k}=\partial \Omega_{k}[21] ; \mathbf{n}_{k}=\left[n_{k, 1} n_{k, 2}\right]^{T}$ is unit normal vector of domain boundary $\Gamma_{k}=\partial \Omega_{k} ; \mathbf{u}_{k}, k=1, \ldots, n_{k}-1$ is the unknown vector of displacements in domain $\Omega_{k} ; \widetilde{B}_{k}^{-}, \widetilde{B}_{k}^{+}, k=2, \ldots, n_{k}-1, \widetilde{B}_{1}^{+}, \widetilde{B}_{n_{k}}^{-}$are matrices (operators) of boundary conditions of the sixth order ( $x_{3}$-independent); $\widetilde{\mathbf{g}}_{k}^{-}, \widetilde{\mathbf{g}}_{k}^{+}, k=2, \ldots, n_{k}-1$, $\tilde{\mathbf{g}}_{1}^{+}, \widetilde{\mathbf{g}}_{n_{k}}^{-}$are right-side vectors of boundary conditions of the sixth order ( $x_{3}$-independent); $\overline{\widetilde{F}}_{k}$ is the right-side vector in domain $\Omega_{k} ; \mathbf{F}_{k}$ is the vector of body forces in domain $\Omega_{k}$; $\mathbf{f}_{k}$ is the boundary traction vector in domain $\Omega_{k} ; \bar{\lambda}_{k}, \bar{\mu}_{k}$ are Lame coefficients of material in domain $\Omega_{k} ; C_{k}$ is the matrix of elastic parameters of the supports (if any); $c_{k, i}$ is the coefficient of resistance in the direction of the axis $O x_{i} ; \partial_{k}=\partial / \partial x_{k}, \partial_{k}^{*}=-\partial / \partial x_{k}, k=1,2$; $\mathbf{v}_{k}=\partial_{2} \mathbf{u}_{k}=\mathbf{u}_{k}^{\prime} ; \mathbf{v}_{k}^{\prime}=\partial_{3} \mathbf{v}_{k}$.

\subsection{Discrete-Continual operational formulation of the problem}

DCFEM presupposes finite element approximation of extended domain along directions of structure perpendicular to the basic direction, while along basic direction problem remain continual (thus extended domain is divided into discrete-continual finite elements). Resultant multipoint boundary problem for the first-order system of ordinary differential equations with piecewise-constant coefficients within DCFEM [14] has the form:

$$
\left\{\begin{array}{l}
\mathbf{y}_{k}^{(1)}-A_{k} \mathbf{y}_{k}=\mathbf{f}_{k}, \quad x_{3} \in\left(x_{3, k}^{b}, x_{3, k+1}^{b}\right), \quad k=1,2, \ldots, n_{k}-1 \\
B_{k}^{-} \mathbf{y}_{k-1}\left(x_{3, k}^{b}-0\right)+B_{k}^{+} \mathbf{y}_{k}\left(x_{3, k}^{b}+0\right)=\mathbf{g}_{k}^{-}+\mathbf{g}_{k}^{+}, \quad k=2, \ldots, n_{k}-1 \\
B_{1}^{+} \mathbf{y}_{1}\left(x_{3,1}^{b}+0\right)+B_{n_{k}}^{-} \mathbf{y}_{n_{k}-1}\left(x_{3, n_{k}}^{b}-0\right)=\mathbf{g}_{1}^{+}+\mathbf{g}_{n_{k}}^{-},
\end{array}\right.
$$

where $N-1$ is the number of discrete-continual finite elements; $A_{k}, k=1,2, \ldots, n_{k}-1$ are matrices of constant coefficients of order $n=4 N$ (discrete analogs of operators $\widetilde{L}_{k}, k=1,2, \ldots, n_{k}-1$ ); $\mathbf{f}_{k}, k=1,2, \ldots, n_{k}-1$ are vectors of size $n=4 N$ (discrete analogs of vector functions $\left.\mathbf{S}_{k}, k=1,2, \ldots, n_{k}-1\right) ; B_{k}^{-}, B_{k}^{+}, k=2, \ldots, n_{k}-1, B_{1}^{+}, B_{n_{k}}^{-}$are matrices of boundary conditions of order $n=4 N$ (discrete analogs of $\widetilde{B}_{k}^{-}, \widetilde{B}_{k}^{+}, k=2, \ldots, n_{k}-1, \widetilde{B}_{1}^{+}$, 
$\left.\widetilde{B}_{n_{k}}^{-}\right) ; \mathbf{g}_{k}^{-}, \mathbf{g}_{k}^{+}, \quad k=2, \ldots, n_{k}-1, \mathbf{g}_{1}^{+}, \mathbf{g}_{n_{k}}^{-}$are right-side vectors of boundary conditions of size $n=4 N$ (discrete analogs of $\widetilde{\mathbf{g}}_{k}^{-}, \widetilde{\mathbf{g}}_{k}^{+}, k=2, \ldots, n_{k}-1, \widetilde{\mathbf{g}}_{1}^{+}, \widetilde{\mathbf{g}}_{n_{k}}^{-}$);

$$
\begin{aligned}
& \mathbf{y}_{k}=\mathbf{y}_{k}\left(x_{2}\right)=\left[\begin{array}{ll}
\mathbf{u}_{k}^{T}\left(x_{2}\right) & \mathbf{v}_{k}^{T}\left(x_{2}\right)
\end{array}\right]^{T} ; \\
& \mathbf{u}_{k}=\mathbf{u}_{k}\left(x_{2}\right)=\left[\left(\mathbf{u}_{n}^{(k, 1)}\right)^{T}\left(\mathbf{u}_{n}^{(k, 2)}\right)^{T} \ldots\left(\mathbf{u}_{n}^{(k, N)}\right)^{T}\right]^{T} ; \\
& \mathbf{v}_{k}=\mathbf{v}_{k}\left(x_{2}\right)=\left[\left(\mathbf{v}_{n}^{(k, 1)}\right)^{T}\left(\mathbf{v}_{n}^{(k, 2)}\right)^{T} \ldots\left(\mathbf{v}_{n}^{(k, N)}\right)^{T}\right]^{T} ; \\
& \mathbf{u}_{n}^{(k, p)}=\mathbf{u}_{n}^{(k, p)}\left(x_{2}\right)=\left[u_{1}^{(k, p)} u_{2}^{(k, p)}\right]^{T}, p=1,2, \ldots, N ; \\
& \mathbf{v}_{n}^{(k, p)}=\mathbf{v}_{n}^{(k, p)}\left(x_{2}\right)=\left[v_{1}^{(k, p)} v_{2}^{(k, p)}\right]^{T}, p=1,2, \ldots, N ;
\end{aligned}
$$

$u_{i}^{(k, p)}=u_{i}^{(k, p)}\left(x_{2}\right), p=1,2, \ldots, N$ are components of displacement $u_{i}$ in the node with coordinate $\left(x_{1}^{p}, x_{2}\right)$ on the interval $x_{2} \in\left(x_{2, k}^{b}, x_{2, k+1}^{b}\right)$.

\section{Analytical solution of resultant multipoint boundary problem}

Solution of problem (6) is accentuated by numerous factors. They include boundary effects (stiff systems) and considerable number of differential equations (several thousands). Moreover, matrices of coefficients of a system normally have eigenvalues of opposite signs and corresponding Jordan matrices are not diagonal. Special method of solution of multipoint boundary problems for systems of ordinary differential equations with piecewise constant coefficients in structural analysis has been developed. Not only does it overcome all difficulties mentioned above but its major peculiarities also include universality, computer-oriented algorithm [22, 23], computational stability, optimal conditionality of resultant systems and partial Jordan decomposition of matrix of coefficient, eliminating necessity of calculation of root (principal) vectors [24].

\subsection{Construction of partial Jordan decomposition}

Jordan decomposition of matrix $A_{k}$ has the form [25-30]

$$
A_{k}=T_{k} J_{k} T_{k}^{-1}, \text { where } J_{k}=\left\{J_{k, 1}, J_{k, 2}, \ldots, J_{k, u_{k}}\right\}
$$

$T_{k}$ is the matrix of order $n$, which columns are eigenvectors and root vectors of matrix $A_{k}$; $J_{k}$ is Jordan matrix of order $n ; J_{k, p}$ is Jordan cell corresponding to eigenvalue $\lambda_{k, p}$; $\operatorname{dim} J_{k, p}=m_{k, p}$.

As we have already mentioned above, specificity of problems of structural analysis comprises in presence of multiple eigenvalues of matrix $A_{k}$ and consequently in necessity of calculation of root vectors. However at the present time there are no effective numerical method of calculation of Jordan decomposition in the general case [23, 24]. Meanwhile the number of multiple eigenvalues in the considering type of problems is normally limited. Besides these multiple eigenvalues are generally zeros. In this connection special alternative approach to solution has been developed. 
Partial Jordan decomposition is based on computation of right and left eigenvectors of matrix $A_{k}$.

$$
A_{k}=A_{k, 1}+A_{k, 2}, \quad \text { where } \quad A_{k, 1}=T_{k, 1} J_{k, 1} \widetilde{T}_{k, 1} ; \quad A_{k, 2}=A_{k}-A_{k, 2}
$$

$T_{k, 1}$ is the matrix containing right eigenvectors corresponding to non-zero eigenvalues of matrix $A_{k} ; \widetilde{T}_{k, 1}$ is the matrix containing left eigenvectors corresponding to non-zero eigenvalues of matrix $A_{k} ; J_{k, 1}$ is diagonal Jordan matrix corresponding to non-zero eigenvalues of matrix $A_{k} ; A_{k, 2}$ is the part of matrix $A_{k}$ corresponding to prime and multiple zero eigenvalues. It is necessary to note here that matrices $T_{k, 1}$ and $\widetilde{T}_{k, 1}$ in general case are rectangular.

\subsection{Construction of fundamental matrix-function of system of equations}

Eigenvalues $\lambda_{k, p}, \quad p=1, \ldots, u_{k}$ are renumbered according to the condition

$$
\left.\begin{array}{ll}
\forall \lambda_{k, p}, & p=1, \ldots, l_{k} \quad \exists m_{k, p}=1 \\
\forall \lambda_{k, p}, & p=l_{k}+1, \ldots, u_{k} \quad \exists m_{k, p}>1
\end{array}\right\} ; \quad l_{k}=\sum_{p=1}^{u_{k}} \delta_{1, m_{k, p}},
$$

where $\delta_{i, j}$ is Kronecker delta.

Due to distinctive procedure, we should properly modify matrices $T_{k, 1}, \widetilde{T}_{k, 1}$ and $J_{k, 1}$.

Let $P_{k, 1}$ and $P_{k, 2}$ be projectors to subspaces of left and right eigenvectors and root vectors of matrix $A_{k}$ corresponding to non-zero and zero eigenvalues. They may be denoted as ( $E$ is identity matrix)

$$
P_{k, 1}=T_{k, 1}\left(\widetilde{T}_{k, 1} T_{k, 1}\right)^{-1} \widetilde{T}_{k, 1} ; \quad P_{k, 2}=E-P_{k, 1} .
$$

After sorting and biorthogonalization of eigenvectors and eigenvalues fundamental matrix-function $\varepsilon_{k}(x)$ of system from (6) for arbitrary $k$ is constructed in the special form convenient for problems of structural mechanics [16]

$$
\varepsilon_{k}(x)=T_{k, 1} \widetilde{\varepsilon}_{k, 0}(x) \widetilde{T}_{k, 1}+\chi(x, 0)\left[P_{k, 2}+\sum_{j=1}^{m_{k, \max }-1} \frac{x^{j}}{j !} A_{k, 2}^{j}\right],
$$

where $\quad m_{k, \max }=\max _{l \leq i \leq u_{k}} m_{k, i} ; \quad \chi\left(x, \lambda_{k, p}\right)=\left\{\begin{array}{l}\operatorname{sign}(x) \theta\left(-\operatorname{Re}\left(\lambda_{k, p}\right) x\right), \quad \lambda_{k, p} \neq 0 \\ 0.5 \cdot \operatorname{sign}(x), \quad \lambda_{k, p}=0 ;\end{array}\right.$

$$
\begin{gathered}
\operatorname{sign}(x)=\left\{\begin{array}{ll}
1, & x>0 \\
-1, & x<0 ;
\end{array} \quad \theta(x)= \begin{cases}1, & x>0 \\
0, & x<0 ;\end{cases} \right. \\
\widetilde{\varepsilon}_{k, 0}(x)=\operatorname{diag}\left\{\chi\left(x, \lambda_{k, 1}\right) \exp \left(\lambda_{k, 1} x\right), \ldots, \quad \chi\left(x, \lambda_{k, l_{k}}\right) \exp \left(\lambda_{k, l_{k}} x\right)\right\} .
\end{gathered}
$$

It should be stated that the sum in the right side of (16) contains four or lower components and corresponds to so-called "beam" part of solution of system. 


\subsection{General solution of the multipoint boundary problem}

Solution of considering problem (6) on the interval $\left(x_{k}^{b}, x_{k+1}^{b}\right)$ is defined by formula

$$
\mathbf{y}_{k}(x)=\left(\varepsilon_{k}\left(x-x_{k}^{b}\right)-\varepsilon_{k}\left(x-x_{k+1}^{b}\right)\right) \mathbf{C}_{k}+\varepsilon_{k} * \mathbf{f}_{k}, \quad x \in\left(x_{k}^{b}, x_{k+1}^{b}\right),
$$

where $\mathbf{C}_{k}$ is the vector of constants of order $n ; *$ is convolution notation [21];

$$
\bar{f}_{k}(x) \equiv f(x) \theta\left(x, x_{k}^{b}, x_{k+1}^{b}\right) ; \quad \theta\left(x, x_{k}^{b}, x_{k+1}^{b}\right)= \begin{cases}1, & x \in\left(x_{k}^{b}, x_{k+1}^{b}\right) \\ 0, & x \notin\left(x_{k}^{b}, x_{k+1}^{b}\right) .\end{cases}
$$

We can rewrite (20) in the form

$$
\begin{gathered}
\mathbf{y}_{k}(x)=E_{k}(x) \mathbf{C}_{k}+\mathbf{S}_{k}, x \in\left(x_{k}^{b}, x_{k+1}^{b}\right) ; \\
E_{k}(x)=\varepsilon_{k}\left(x-x_{k}^{b}\right)-\varepsilon_{k}\left(x-x_{k+1}^{b}\right) ; \quad \mathbf{S}_{k}(x)=\varepsilon_{k} * \mathbf{f}_{k} .
\end{gathered}
$$

Substituting (22) in boundary conditions (6) and taking into account that

$$
\begin{gathered}
E_{k-1}\left(x_{k}^{b}-0\right)=\varepsilon_{k-1}\left(h_{k-1}^{b}\right)-\varepsilon_{k-1}(-0), \quad k=2, \ldots, n_{k} ; \quad h_{k}^{b}=x_{k+1}^{b}-x_{k}^{b}, \quad k=1, \ldots, n_{k}-1 ; \\
E_{k}\left(x_{k}^{b}+0\right)=\varepsilon_{k}(+0)-\varepsilon_{k}\left(-h_{k}^{b}\right), \quad k=1, \ldots, n_{k}-1 ;
\end{gathered}
$$

we have the following system of linear algebraic equations for $\mathbf{C}_{k}, k=1, \ldots, n_{k}-1$

$$
K \mathbf{C}=\mathbf{G},
$$

where matrix $K$ can be divided into so-called main $K^{0}$ and additional $K^{1}$ members,

$$
\begin{aligned}
& K^{0}=\left[\begin{array}{cccccc}
K_{1,1}^{0} & 0 & 0 & . . & 0 & K_{1, n_{k}-1}^{0} \\
K_{2,1}^{0} & K_{2,2}^{0} & 0 & . . & 0 & 0 \\
0 & K_{3,2}^{0} & K_{3,3}^{0} & \ldots & 0 & 0 \\
\ldots & \ldots & \ldots & \ldots & \ldots & \ldots \\
0 & 0 & 0 & . . & K_{n_{k}-1, n_{k}-2}^{0} & K_{n_{k}-1, n_{k}-1}^{0}
\end{array}\right] ; \\
& K^{1}=\left[\begin{array}{cccccc}
K_{1,1}^{1} & 0 & 0 & . . & 0 & K_{1, n_{k}-1}^{1} \\
K_{2,1}^{1} & K_{2,2}^{1} & 0 & . . & 0 & 0 \\
0 & K_{3,2}^{1} & K_{3,3}^{1} & \ldots & 0 & 0 \\
\ldots & \ldots & \ldots & \ldots & \ldots & \ldots \\
0 & 0 & 0 & . . & K_{n_{k}-1, n_{k}-2}^{1} & K_{n_{k}-1, n_{k}-1}^{1}
\end{array}\right] ; \\
& K_{k, k-1}^{0}=-B_{k}^{-} \varepsilon_{k-1}(-0) ; \quad K_{k, k}^{0}=B_{k}^{+} \varepsilon_{k}(+0) ; \quad K_{1,1}^{0}=B_{1}^{+} \varepsilon_{1}(+0) ; \quad K_{1, n_{k}-1}^{0}=-B_{n_{k}}^{-} \varepsilon_{n_{k}-1}(-0) ; \\
& K_{k, k-1}^{1}=B_{k}^{-} \varepsilon_{k-1}\left(h_{k-1}^{b}\right) ; K_{k, k}^{1}=-B_{k}^{+} \varepsilon_{k}\left(-h_{k}^{b}\right) ; K_{1,1}^{1}=-B_{1}^{+} \varepsilon_{1}\left(-h_{1}^{\mathrm{b}}\right) ; K_{1, n_{k}-1}^{1}=B_{n_{k}}^{-} \varepsilon_{n_{k}-1}\left(h_{n_{k}-1}^{b}\right) ;(30) \\
& \mathbf{G}=\left[\begin{array}{llll}
\mathbf{G}_{1}^{T} & \mathbf{G}_{2}^{T} & \ldots & \mathbf{G}_{n_{k}-1}^{T}
\end{array}\right]^{T} ; \quad \mathbf{C}=\left[\begin{array}{llll}
\mathbf{C}_{1}^{T} & \mathbf{C}_{2}^{T} & \ldots & \mathbf{C}_{n_{k}-1}^{T}
\end{array}\right]^{T} ;
\end{aligned}
$$




$$
\begin{gathered}
\mathbf{G}_{1}=\mathbf{g}_{1}^{+}+\mathbf{g}_{n_{k}}^{-}-B_{1}^{+} \mathbf{S}_{1}\left(x_{1}^{b}+0\right)-B_{n_{k}}^{-} \mathbf{S}_{n_{k}-1}\left(x_{n_{k}}^{b}-0\right) ; \\
\mathbf{G}_{k}=\mathbf{g}_{k}^{-}+\mathbf{g}_{k}^{+}-B_{k}^{-} \mathbf{S}_{k-1}\left(x_{k}^{b}-0\right)-B_{k}^{+} \mathbf{S}_{k}\left(x_{k}^{b}+0\right), k=2, \ldots, n_{k}-1 .
\end{gathered}
$$

Symbol $\otimes$ imply direct product of matrices. It is necessary to note that matrices $\varepsilon_{k}(+0)$ and $\varepsilon_{k}(-0)$ are independent of $x$.

We find it vital to note that diagonal blocks of matrix $K$ are practically singular. This fact leads to several problems. Iterative methods of solution can't be applied in particular. Gaussian elimination method with pivoting is required. It is useful to specify ways of disposal of this disadvantage.

Let us transform considering system of equation as follows: each equation of system, since the first (and finishing next to last), we will replace with the sum of this equation with the subsequent (instead of the initial first equation we take the sum initial the first with initial the second, instead of initial the second - the sum initial the second with initial the third and so on). Instead of initial last equation we take the sum of the initial last with initial the first. Finally we removed the singularity mentioned above. Finally we have:

$$
K=\left[\begin{array}{cccccccc}
\widetilde{K}_{1,1} & \widetilde{K}_{1,2} & 0 & 0 & . . & 0 & 0 & \widetilde{K}_{1, n_{k}-1} \\
\widetilde{K}_{2,1} & \widetilde{K}_{2,2} & \widetilde{K}_{2,3} & 0 & . . & 0 & 0 & 0 \\
0 & \widetilde{K}_{3,2} & \widetilde{K}_{3,3} & \widetilde{K}_{3,4} & \ldots & 0 & 0 & 0 \\
\ldots & \ldots & \ldots & \cdots & \ldots & \ldots & \ldots & \ldots \\
\widetilde{K}_{n_{k}-1,1} & 0 & 0 & 0 & . . & 0 & \widetilde{K}_{n_{k}-1, n_{k}-2} & \widetilde{K}_{n_{k}-1, n_{k}-1}
\end{array}\right] .
$$

The Reported study was Funded by Government Program of the Russian Federation "Development of science and technology" (2013-2020) with in Program of Fundamental Researches of Ministry of Construction, Housing and Utilities of the Russian Federation and Russian Academy of Architecture and Construction Sciences, the Research Project 7.1.1.

\section{Conclusions}

Development, research and verification of correct mathematical models and methods of structural mechanics are the most important aspects of ensuring safety of buildings and complexes. The field of application of discrete-continual finite element method (DCFEM) comprises structures with regular (in particular, constant or piecewise constant) physical and geometrical parameters in some dimension (so-called "basic" direction (dimension)). Considering problems remain continual along "basic" direction while along other directions DCFEM presupposes mesh approximation. DCFEM has completely computer-oriented algorithm, computational stability, optimal conditionality of resultant system and applicable for the various loads at an arbitrary point or a region of the shear wall. This method allows increasing the accuracy of the solution (especially in solution of high-rise shear walls) and provides reduction of the computational efforts.

\section{References}

1. O.C. Zienkiewicz, R.L. Taylor, The finite element method: Solid mechanics. Vol. 2 (Butterworth-heinemann, 2014) 
2. R. LeVeque, Finite Difference Methods for Ordinary and Partial Differential Equations: Steady-State and Time-Dependent Problems (Classics in Applied Mathematics, SIAM, 2007)

3. S.A. Sauter, C. Schwab, Boundary element methods (Springer Berlin Heidelberg, 2011)

4. J. Kevorkian, Partial differential equations: Analytical solution techniques (Springer, 2000)

5. A.B. Zolotov, P.A. Akimov, V.N. Sidorov, M.L. Mozgaleva, Numerical and analytical methods for design of structures (ASV Publishing House, 2009)

6. W.-X. Zhong, X.-X. Zhong, Computers \& Structures 37(6), 993-1004 (1990)

7. A.B. Zolotov, P.A. Akimov, M.L. Mozgaleva, Multilevel discrete and discretecontinual variational-difference methods (ASV Publishing House, 2013)

8. P.A. Akimov, M.L. Mozgaleva, Applied Mechanics and Materials 351-352, 13-16 (2013)

9. P.A. Akimov, M.L. Mozgaleva, Applied Mechanics and Materials 405-408, 3165-3168 (2013)

10. P.A. Akimov, M.L. Mozgaleva, M. Aslami, O.A. Negrozov, Applied Mechanics and Materials 670-671, 720-723, (2014)

11. P.A. Akimov, M.L. Mozgaleva, M. Aslami, O.A. Negrozov, Applied Mechanics and Materials 670-671, 724-727, (2014)

12. U.M. Ascher, R.M. Mattheij, R.D. Russell, Numerical Solution of Boundary Value Problems for Ordinary Differential Equations (Classics in Applied Mathematics, SIAM, 1995).

13. I.M. Smith, Programming the Finite Element Method (John Wiley \& Sons Ltd, 2004)

14. P.A. Akimov, A.M. Belostosky, M.L. Mozgaleva, M. Aslami, O.A. Negrozov, Advanced Materials Research 1040, 664-669 (2014)

15. M.H. Sadd, Elasticity: theory, applications, and numerics (Academic Press, 2014)

16. A.B. Zolotov, P.A. Akimov, V.N. Sidorov, M.L. Mozgaleva, Mathematical Methods in Structural Mechanics (ASV Publishing House, 2008)

17. R.T. Malone, R. Rice, The Analysis of Irregular Shaped Structures Diaphragms and Shear Walls (McGraw Hill Professional, 2011)

18. M.Y.H. Bangash, T. Bangash, Elements of spatial structures: analysis and design (Thomas Telford, 2003)

19. D.S. Chandrasekharaiah, L. Debnath, Continuum mechanics (Elsevier, 2014).

20. P.A. Akimov, M.L. Mozgaleva, Applied Mechanics and Materials 580-583, 2898-2902 (2014)

21. P. Teodorescu, W.W. Kecs, A. Toma, Distribution Theory: With Applications in Engineering and Physics (John Wiley \& Sons, 2013)

22. S. Blair-Chappell, A. Stokes, Parallel Programming with Intel Parallel Studio XE (John Wiley \& Sons, 2012)

23. M. Metcalf, J. Reid, M. Cohen, Modern FORTRAN Explained (Numerical Mathematics and Scientific Computation) (Oxford University Press, 2011)

24. P.A. Akimov, V.N. Sidorov, Advanced Materials Research 250, 3652-3655 (2011)

25. R. Piziak, P.L. Odell, Matrix theory: From generalized inverses to Jordan form (Chapman and Hall/CRC, 2007)

26. S.H. Weintraub, Synthesis Lectures on Mathematics and Statistics 2(1), 1-108 (2009)

27. J.N. Franklin, Matrix theory (Courier Corporation, 2012).

28. F. Zhang, Matrix theory: basic results and techniques (Springer Science \& Business Media, 2011).

29. A. Bjorck, Numerical methods in matrix computations (Springer, 2015).

30. D. Poole, Linear algebra: A modern introduction (Cengage Learning, 2014). 